\title{
Schneller?! Zeit und Medizin
}

\author{
T. Lempert
}

Das Phänomen Zeit umfasst viele Facetten. Die naheliegendste und wohl wichtigste ist der Medizin ins Herz geschrieben. Wir Menschen sind endlich, unsere Zeit auf der Welt ist begrenzt. Welche andere Berufsgruppe wie die der Ärztinnen und Ärzte ist in der Lage, die Lebenszeit zu verlängern, also Zeit zu schenken? Also sollte man meinen, es besteht auf allen Seiten uneingeschränkte Akzeptanz einer Meinungsführerschaft der Medizin in Belangen der Zeit. Aber im gesellschaftlichen Meinungsbildungsprozess um lebensverlängernde medizinische Massnahmen etwa finden sich kaum medizinische Fachleute. Die Politikerin diskutiert, der Theologe ist dabei, Philosophen lassen sich in den Diskussionen ausmachen. Wo aber stecken die Ärztinnen und Ärzte (neben der Teilnahme an internen Meinungszirkeln)?

\section{Zeitmanagementtips beiseitelegen}

Wenden wir uns weniger solch brisant medizinethischen Themen zu? Woran liegt es, dass die Standardrezepte der Zeitmanagementratgeber im Bereich der Medizin häufig zu kurz greifen? Im konkreten medizinischen Tagesgeschäft, der Arbeit an der Patientin, lässt sich etwa das Postulat eines exakt vordefinierten zeitlichen Rahmens eines Arbeitsschrittes nicht eins zu eins übertragen. Noch immer geht es um Menschen und die Komplexität ihrer biologischen Funktionen, da kann der Bleistift nicht auf die Minute fallengelassen werden. Da lauert eine Falltür in der interdisziplinären Diskussion. Waren in früheren Zeiten die beiden wichtigsten Akteure im Spital - die Pflegefachpersonen und die Ärztinnen und Ärzte - zeitlich (fast) uneingeschränkt für die Patientin da, haben sich die Pflegenden längst aus dieser Allianz verabschiedet. Wer also eine Patientin nach Ablauf der persönlichen Arbeitszeit noch untersucht, ist die Ärztin. Wie aber gehen die Mediziner mit dieser unerfreulichen Tatsache um? Wohl nur sehr vorsichtig murrend, statt laut ausrufend. Dringend ist hier ein Dialog erforderlich, der nicht auf einseitigen Massnahmen beruht und die andere Seite vor vollendete Tatsachen stellt. Das Angebot zum Dialog über den konkreten Umgang mit begrenzten zeitlichen Arbeitsressourcen in Spitalorgani- sationen sollte von den Medizinerinnen und Medizinern kommen, wenn die Ärzte zumindest die ideelle Führung des Spitalwesens auch weiterhin behalten wollen (oder wiedergewinnen möchten).

Aber zu Recht wird in einem solchen Dialog darauf hingewiesen werden, dass die einzelne Ärztin selbst zu einer angemessenen Form des Umgangs mit der Zeit finden muss. Noch immer sind Zuspätkommende bei Sitzungen oder Visiten im Spital Ärzte; und selten lassen sich von allen akzeptierte Entschuldigungen heranziehen, denn nicht jedes Zuspätkommen hat mit einer Notsituation am Bett zu tun.

\section{Entscheidungen klar treffen}

Warum leben viele Mediziner mit ihrem Terminkalender auf Kriegsfuss? Neben dem bedauerlichen Umstand, dass es Mediziner gibt, die gar keinen Terminkalender besitzen oder wenn, ihn dann durch das Sekretariat fremdbestimmen lassen, kann auch folgende Ursache ausgemacht werden: Im Zuge des zeitgeistigen Mitbestimmungsverständnisses gibt es zu viele herausgeschobene Entscheidungen oder Lösungsvorschläge, die erst nach endlosen Diskussionen gefasst werden. Es fehlt vielen Medizinern schlicht der Mut und auch die Übung, einfach ja oder nein zu sagen und eine dezidierte, auch schnelle Wahl aus den - meist - verschiedenen Möglichkeiten des medizinischen Handelns zu treffen (lieber bei heiklen Fragen in ein «board» damit). Da ist Unsicherheit und die Angst auszumachen, zur eigenen Entscheidung zu stehen - sei es vor der Chefärztin, vor dem Oberarzt, vor der Pflegefachfrau oder auch vor dem belesenen Angehörigen.

Entscheidungen treffen heisst zudem immer auch, Selbstbestimmung verwirklichen. Das Gefühl zu haben, Meister und Meisterin des eigenen Tuns zu sein, bringt Befriedigung. Und das sollte bei der Arbeit nicht mit dem schamhaften Tuch etwaiger Anmassung oder egoistischem Einzelgängertum verhängt sein. Eine Förderung von durchaus nötigem Gemeinschaftsgeist sollte auch vor der Sichtung der Differenzen und der Profile der Beteiligten nicht haltmachen. 


\section{Leistung durch Schnelligkeit}

Des weiteren gibt es sehr persönliche Gründe, Zeitgestaltung bei der Arbeit als entscheidend wichtig anzuschauen. Es ist natürlich nicht alles schneller und damit vermeintlich effizienter zu machen (und über Langzeitfolgeschäden des Schnelligkeitswahns spricht der Versicherungsdirektor ungern). Trotzdem: Diese tiefe Befriedigung, in kurzer Zeit viel und zudem äusserst Sinnvolles geleistet zu haben! Welche Ärztin, welcher Arzt kennt dieses Gefühl nicht? Das gibt Kraft und Selbstvertrauen. Das Gefühl der befriedigenden Schnelligkeit und damit Leistungsfähigkeit ist heute eher anrüchig. Das positiv erfahrene persönliche «Aufgehen» in einer spezifischen Arbeitssituation nämlich ist ein entscheidender Aspekt in der Arbeitsglücksforschung, bei dem sogenannten flow-Erlebnis. Welche andere Berufsgruppe hat schon diese berufliche Eigenschaft, immer wieder Zufriedenheit innerhalb kurzer Zeitfenster zu erreichen? Dieser Aspekt des Verständnisses von Zeit kann auch in die Waagschale der ärztlichen (Zeit-)Erfahrung gelegt werden.

Was also bringt positive Arbeitskraft in Bewegung: diese Frage muss heute gestellt werden, ohne dass die Antwort unverzüglich den Ruf nach Wellness für Angestellte mit sich bringt.

Differenzierungsfähigkeit ist somit eine Eigenschaft, die vom ärztlichen Berufsstand auch beim
Thema Zeit und Zeitmanagement gefordert werden kann. Eine klare Meinung bezüglich Nutzen und Unsinn der Zeitmanagementliteratur für das Gesundheitswesen ist in der Diskussion mit den anderen Berufsständen erforderlich.

Wenn bei einem «effizienten», sinnvollen persönlichen Zeitmanagement tatsächlich Zeit eingespart wird, dient diese Zeiteinsparung oft nur dem Erfüllen neuer Aufgaben und keineswegs dem Etablieren (auch) nötiger Ruhepausen. Hier wird es wichtig, selbstbewusst auch aus medizinischen Gründen dem Raubbau an der eigenen Gesundheit entgegenzutreten; nötigenfalls neben dem gemeinschaftlichen aktiven Widerstand gegen immer neue (Verwaltungs-)Aufgaben auch mit einem persönlichen passiven, nämlich mit dem Entsorgen von überflüssigen Unterlagen in den Papierkorb.

Das Einfordern von zuallererst der Kenntnis um persönliche Zeitressourcen und dann dem bewussten Umgang damit dient zuletzt auch der Gesundheitsprophylaxe, als Schutz vor einem lauernden Burn-out nämlich.

\section{Literatur}

- $\quad$ Baeriswyl M. Chillout. Wege in eine neue Zeitkultur. München: dtv; 2000.

- Csikszentmihalyi M. Das flow-Erlebnis. Stuttgart: Klett-Cotta; 1985. 


\section{Schneller!? Sinnvolles Zeitmanagement in der Medizin}

\section{«Speed is God and time is the Devil»}

Zeitdruck ist ein Dauerthema in der Medizin (und anderswo). Mit den aktuellen gesellschaftlichen und politischen Entwicklungen zeichnen sich nur weitere Verschärfungen ab. «Es gibt keinen Hinweis darauf, dass irgendetwas langsamer werden wird.»

Die diversen Bestseller der Zeitmanagementliteratur und -beratung liefern einige sinnvolle Hinweise zur Verbesserung des eigenen Umgangs mit Zeit - und doch greifen diese Rezepte häufig zu kurz. Zudem sind sie nicht auf die Arbeitssituationen und die Beschäftigten des Gesundheitssystems bezogen.

In diesem Angebot werden die aktuellen Zeitmanagementkonzepte deshalb speziell auf ihren Nutzen in einem Spitalbetrieb überprüft und mit neuen Konzepten aus anderen Bereichen ergänzt (work-life-balance, flow, Glücksforschung, Sinnhaftigkeit des Tuns, Selbstbestimmungsmöglichkeiten, Entspannung und Leistungsfähigkeit, Burn-out-Prophylaxe usw.).

Das Ziel ist es, den Teilnehmenden durch ein umfassendes Modell der Gestaltung der eigenen Arbeit Möglichkeiten anzubieten, wie sie mit Zeitdruck, aber auch mit anderen Stressoren ihrer Arbeit so umgehen können, dass die Arbeit gleichzeitig effektiv und befriedigend gestaltet werden kann.

\section{Zielgruppe}

Ärzte.

\section{Programmleiter}

- Thomas Lempert, lic. theol., CAGS, Beratungen und Coaching von Führungskräften und Teams, Dozent und Lehrtherapeut

- Hansjürg Lusti, Dipl. Psychologe IAP, Supervisor und Organisationsberater BSO, Systemtherapeut SGS

\section{Daten und Ort}

Donnerstag, 15. September 2005 College-M

\section{Preis}

Fr. 350.-, darin enthalten sind sämtliche Programmaktivitäten und Unterlagen.

\section{Informationen und Anmeldungen}

Detaillierte Informationen finden Sie unter: www.college-m.ch oder erhalten Sie durch: College-M, Freiburgstrasse 41, 3010 Bern, Tel. 03163230 26, Fax 03163230 25, E-Mail: info@college-m.ch. 